\title{
Dummy Chest Pressure Experiment Parameters Optimal Selection and Optimization Method Based on FEM
}

\author{
Kai Ma ${ }^{1,2, a}$, Tuan Wang ${ }^{* 1,1, a}$, Xin $\mathrm{Lu}^{1, a}$, Ji-dong Gao ${ }^{2, a}$, Tao Xu ${ }^{1, a}$ \\ ${ }^{1}$ Institute of Mechanical Science and Engineering, Jilin University ,Changchun, China, 130022 \\ ${ }^{2}$ China Automotive Technology \&Research Center ,Automotive Engineering Research Institute, \\ Tianjin ,China,300300
}

a1263184355@qq.com

\begin{abstract}
Keywords: vehicle collision FEM dummy parameter optimization parameter optimal selection
Abstract. Based on vehicle collision test, a finite element dummy model (FEM dummy) was built, at the same time the parameter optimization method was proposed in the paper. To improve consistency between the dummy simulation results and results of vehicle collision test, it's necessary to scientifically adjust FEM dummy's material parameters. First, parameter optimal selection applied, the most important parameters were selected. Then, using optimal methods obtained FEM dummy correction result which is similar to that of the real vehicle. Finally, chest pressure result curve of a FEM dummy model in positive collision as an example, main materials parameters of the FEM dummy's chest were selected by parameter optimal selection, then the parameters were modified by optimization method. ensuring simulation dummy' chest pressure peaks and spring rate are more consistent with vehicle collision result. It further found that the proposed method could be used to improve the FEM dummy model to meet the result of the vehicle collision simulation.
\end{abstract}

\section{Introduction.}

In the full-size vehicle collision, the dummy simulation is essential. About the research and development of the finite element model of the positive impact dummy, foreign experts have accumulated certain experience. There are several main types of frontal crash dummies internationally: 50th percentile adult male dummy, 95th percentile adult male dummy, 5 percentile female dummy, Infants under one year old, 3-year-old, 13-year-old children, the elderly pregnant women and so on. Currently, the world largest car companies widely use the finite element model of the positive impact dummy, which is mainly produced and offered by the FTSS (first technology safely systems) and Germany's DYNAmore company. Foreign company's positive crash dummy products have achieved series, commercialization and standardization. The main direction of domestic research for positive crash dummy is zooming positive crash dummy to meet the Chinese human dimensions.

Based on Chinese human dimensions, Lin daquan et al. completed the manufacture of Chinese 95 percentile crash dummy use finite element analysis method. To find out the development program for crash dummy fitting the Chinese 50th percentile human, Cao libo et al. proposed method through the part of the body section zooming. On the practical engineering based on a new Chinese FEM dummy, we aim to improve the accuracy of simulation model. To improve consistency between the dummy simulation results and results of full-size vehicle collision test, it's necessary to reasonably adjust FEM dummy's major structural material parameters and parameter curves. The approximate parameter optimization design method applied to meet this requirement. To illustrate the application of the method, chest pressure result curve of some FEM dummy model in positive collision as an example, some material parameters were optimization designed. The results prove it that the proposed method could be used to improve the FEM dummy model to meet the result of the full-size vehicle collision simulation accuracy. 
In this paper, based on matrix perturbation theory and generalized inverse matrix theory, we will discuss the issues of selecting main structural parameters of the new FEM dummy' chest using the information provided by the sensitivity analysis. According to its root mean square value, we get the design variables modifier which makes the characteristics deviation minimal. And it proves that according to the method of selection to modify $\mathrm{k}$ design parameters, we can make the mean square value' mathematical expectation of the characteristics deviation minimize, and further give expression of the minimum value. Accordingly, based on the ranking method of optimal parameter, this paper propose two kinds of different parameters selection method, the first one is not limited to the number of parameters, the another one is defining the number of parameters, that are the ideal modified parameters selection method and the optimum modification parameters selection method. First, applying the ideal modified parameters selection method to get the most reasonable but the least optimal parameter. Then, using the optimum modification parameters selection method to finally determine optimal parameter according to the number of parameters required. The simulation results show that this method can effectively guarantee the optimization results.

\section{The ideal modified parameters selection method}

Assuming the $b_{1}, b_{2}, \mathrm{~K} b_{m}$ are the design parameters of the structure, and vector $\Delta \bar{w}=\left(\Delta \overline{w_{1}}, \Delta \overline{w_{2}}, \mathrm{~K}, \Delta \bar{w}_{n}\right)^{T}$ is the error vector of the first $n$ frequencies, the sensitivity matrix of the first $\mathrm{n}$ frequencies with respect to all of the design parameters is a $n \times m$ matrix, denoted by $\bar{U}$,

$$
\bar{U}=\left[u_{1}, u_{2}, \mathrm{~K}, u_{m}\right]_{n \times m}
$$

In order to correct the frequency error, $\Delta \bar{w}$, the design parameters of the structure need to be modified. Now assuming that we choose $k(k \leq m)$ parameters from all of $m$ design parameters to be modified, denoted by $b_{i 1}, b_{i 2}, \mathrm{~K}, b_{i k}$, the matrix can be expressed as $b_{i}=\left[b_{i 1}, b_{i 2}, \mathrm{~K}, b_{i k}\right]$, corresponding to those parameters is denoted by $U$, as

$$
U=\left[b_{i 1}, b_{i 2}, \mathrm{~K}, b_{i k}\right]_{n \times k}
$$

According to the perturbation method, when the modification of design parameters $b_{i 1}, b_{i 2}, \mathrm{~K}, b_{i k}$ is $\Delta b_{i}=\left[\Delta b_{i 1}, \Delta b_{i 2}, \mathrm{~K}, \Delta b_{i k}\right]^{T}$, the counterpart of the first $n$ frequencies, $\Delta w$, is given by

$$
\Delta w=U g b
$$

So the corrected for frequency error vector becomes $\psi=\Delta w+\Delta \bar{w}=\Delta w+U g \Delta b$

The root-mean-square of the frequency error vector is given by

$$
\psi_{r m s}^{2}=\psi^{T} \psi=(\Delta w+U g \Delta b)^{T}(\Delta w+U g \Delta b)
$$

Our aim is to find the modification of the parameters $b_{i 1}, b_{i 2}, \mathrm{~K}, b_{i k}$, such that $\psi_{r m s}^{2}$ takes its minimal value. For this purpose, we differentiate the right side of eqn (5) with respect to $\Delta b$ and let it equal to zero, we have

$$
U \mathrm{~g} b=-\Delta \bar{w}
$$

Then we can get the optimal modification of the $\Delta b$, denoted by $\Delta b=U^{+} \Delta \bar{w}$

Where $U^{+}$is the generalized inverse matrix of Moore-Penrose, and can be calculated as

$$
U^{+}=U_{2}^{T}\left(U_{2} U_{2}^{T}\right)^{-1}\left(U_{1}^{T} U_{1}\right)^{-1} U_{1}^{T}
$$

The maximal rank decompositions of matrix $U$ are $U_{1}$ and $U_{2}$, that is, matrices $U_{1}$ and $U_{2}$ satisfy

$$
U=U_{1} \mathrm{~g}_{2}
$$

Where $U_{1}$ is a $n \times r$ matrix and $r$ is its rank, $U_{2}$ is a $r \times k$ matrix and $r$ is its rank, $r=\operatorname{rank}(U)$, is the rank of sensitivity matrix $U$.Substituting eqn(7)into eqn(5),using the properties of generalized 
inverse and eqn(8)to calculate the r.m.s of the corrected frequency errors after the structure is modified, denoted by

$$
\psi_{r m s}^{2}=\Delta \bar{w}_{r m s}^{2}-\Delta \bar{w}^{T} U_{1}\left(U_{1}^{T} U_{1}\right)^{-1} U_{1}^{T} \Delta \bar{w}
$$

The frequency error vector is often a random vector. Denote $C_{\Delta \bar{w} \Delta \bar{w}}$ is its covariance matrix, the expectation of

$$
E\left(\Delta \bar{w}_{r m s}^{2}\right)=E\left(\Delta \bar{w}^{T} g \bar{w}\right)=\sum_{i=1}^{n}\left(C_{\Delta \bar{w} \Delta \bar{w}}\right)_{i i}
$$

Where $E$ is the expectation of random variable. The diagonal terms of $C_{\Delta \bar{w} \Delta \bar{w}}$, denoted by $\left(C_{\Delta \bar{w} \Delta \bar{w}}\right)_{i i}$, are the square of the standard deviations of the $\Delta \overline{w_{i}}$, while the off-diagonal terms measure the correlation between them.

For simplicity, we introduce a random vector $\quad \varphi=L_{r \times r}^{T} U_{1}^{T} \Delta \bar{w}$

Where $L_{r \times r}^{T}$ is a square factor of $\left(U_{1}^{T} U_{1}\right)^{-1}$ e.g. the Cholesky factor, $\left(U_{1}^{T} U_{1}\right)^{-1}=L g^{T}$

The covariance matrix of $\varphi$ is then given as $\quad C_{\varphi \varphi}=E\left(\varphi \varphi^{T}\right)=L^{T} U_{1}^{T} C_{\Delta \bar{w} \bar{w}} U_{1} L$ (14)

From eqn(10), the expected value of $\psi_{r m s}^{2}$ can be calculated as

$$
E\left(\psi_{r m s}^{2}\right)=\sum_{i=1}^{n}\left(C_{\Delta \bar{w} \bar{w}}\right)-\sum_{i i}^{n}\left(C_{\varphi \varphi}\right)_{i i}
$$

\section{The optimum modification parameters selection method}

It can be seen from eqn(15) that we choose $k(k \leq m)$ parameters from all of $m$ design parameters to be modified, denoted by $b_{i 1}, b_{i 2}, \mathrm{~K}, b_{i k}$, so that $E\left(\psi_{r m s}^{2}\right)$ after correction is minimal and the trace of $C_{\varphi \varphi}$ is maximal. Denote

$$
\vartheta=U_{1} L
$$

The columns of $\mathscr{C}^{\mathrm{are}}$ orthonormal vector, then the eqn(14)becomes

$$
C_{\varphi \varphi}=\vartheta^{T} C_{\Delta \bar{w} \Delta \bar{w}} \theta
$$

The eigenvalues and eigenvector corresponding of $C_{\Delta \bar{w} \Delta \bar{w}}$ can be denoted by

$$
0<\lambda_{1} \leq \lambda_{2} \leq \mathrm{K} \lambda_{n} \text { 和 } a_{1}, a_{2}, \mathrm{~K}, a_{n}
$$

Assuming they are satisfied with the orthonormality condition. The corresponding eigenvectors of $\mathrm{r}$ the biggest characteristic value constitute a $n \times r$ matrix, denote

$$
A_{n \times r}=\left[a_{n-r+1}, \mathrm{~K}, a_{n}\right]
$$

Assuming that make the following matrix equation has solutions

$$
A X=\vartheta
$$

$X$ is a $n \times r$ orthogonal matrix, can be calculated as

$$
\sum_{i=1}^{n}\left(C_{\varphi \varphi}\right)=\sum_{j=n-r+1}^{n} \lambda_{j}
$$

Equations (21)-(22)show that if we can choose parameters $b_{i 1}, b_{i 2}, \mathrm{~K}, b_{i k}$, from all of the design parameters such that the rank of their corresponding sensitivity matrix $U, r=\operatorname{rank}(U)=\min (k, \operatorname{rank}(\bar{U}))$, the column vectors of $U$ can be expressed by $a_{n-r+1}, \mathrm{~K}, a_{n}$, then 
$\sum_{i=1}^{r}\left(C_{\varphi \varphi}\right)$ takes its maximum value $\sum_{j=n-r+1}^{r} \lambda_{j}$, the expected value of $\psi_{r m s}^{2}$ gets its minimum value $\sum_{j=1}^{n-r} \lambda_{j}, b_{i 1}, b_{i 2}, \mathrm{~K}, b_{i k}$ is called the ideal modified parameters .

\section{Multi-objective optimization}

This article uses a multi- objective optimization method, this method is a kind of liner multi-objective iterative approximation optimization method, and has been widely used in engineering. The main steps are as follows;

Step 1:seting a minimum $\Delta x$, differently calculating the sensitivity of the function.

$$
d f_{k} / d x_{i}=\frac{f_{k}\left(x_{1}, x_{2}, \mathrm{~L}, x_{i}+\Delta x, \mathrm{~L}, x_{n}\right)-f_{k}(X)}{\Delta x}
$$

Step 2:calculate

$$
\frac{d f_{j} / d x_{i}}{d f_{k} / d x_{i}} \quad k, j=1,2, \mathrm{~L} m \quad i=1,2, \mathrm{~L}, n
$$

Step 3:use the following equations for $\lambda_{1}, \lambda_{2}, \mathrm{~L}, \lambda_{m}$

Step 4:

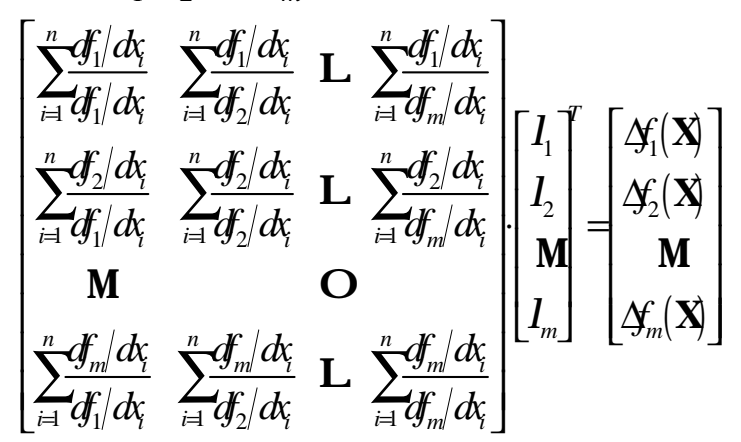

Step 5:

$$
\Delta x_{i}=\lambda_{1} \frac{d f_{1}}{d x_{i}}+\lambda_{2} \frac{d f_{2}}{d x_{i}}+\lambda_{3} \frac{d f_{3}}{d x_{i}}
$$

$$
\Delta f_{k}(\mathbf{X})=f_{k}(\mathbf{X}+\Delta \mathbf{X})-f_{k}(\mathbf{X})
$$

If $\left\|\Delta f_{k}(\mathbf{X})-\Delta f_{k \max }(\mathbf{X})\right\|_{f} \leq \varepsilon$, stop counting; otherwise

$$
d_{k} / d x_{i}=\frac{f_{k}\left(x_{1}, x_{2}, \mathrm{~L}, x_{i}+\Delta_{i}+\Delta x, \mathrm{~L}, x_{n}\right)-f_{k}(X)}{\Delta x}
$$

Back to the second step.

Here $\varepsilon$ is a given threshold.

The method of calculation process is shown in figure1. 


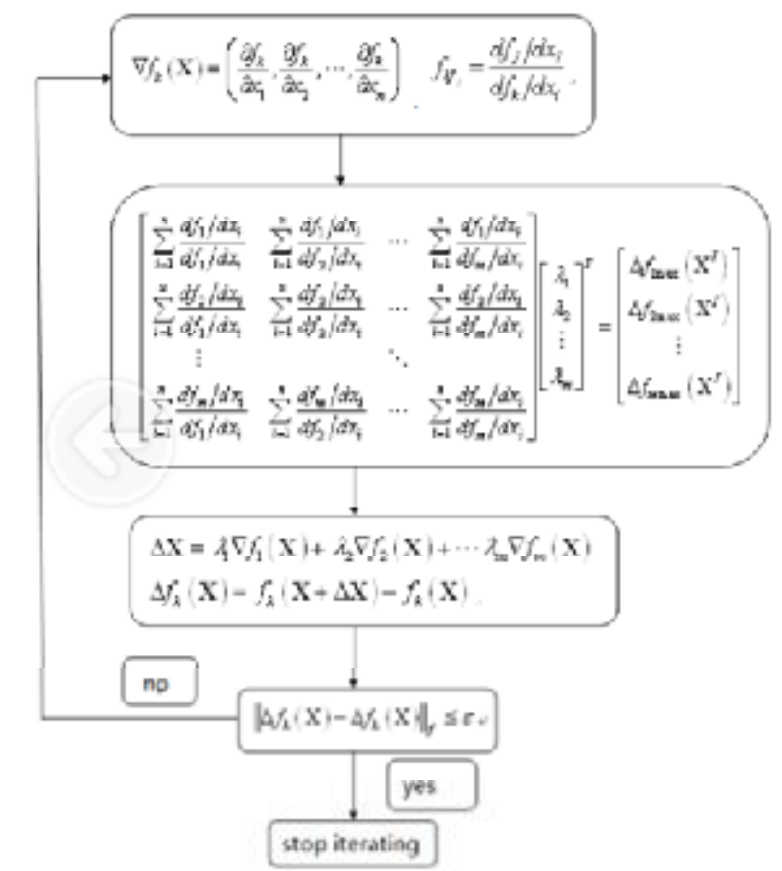

Fig1.Multi-objective optimization program flow ch

\section{Based on Dummy Chest Pressure Experiment the Material Parameters Optimal Selection and Optimization Method}

Based on the rigid pendulum impact experiment of a certain type of finite element dummy chest, this paper improves the main effect of the optimized parameters referring to the results of real dummy model test, and adjusts the compression deformation and rebound rate of the chest reasonably.

Frontal crash dummy model is a parameterized finite element model, as shown in Figure 2 and Figure 3.Compared with the actual crash dummies, the initial model has a certain deviation in such aspects as chest pressure curve. So, adjusting the parameters of dummy model is a very important work. Because of the large number of parameters and the target value, developing a reasonable method is needed. This method should filter out the parameters that affect the outcome of the target remarkably. And it also can determine the amount of modification of these parameters. So we can use the method mentioned above. The main steps are as follows:

Step1: Using the parameter optimization method to select the ideal parameters that meet the conditions from the existing parameters, as shown in formula (1-21).

Step2: Using multi-objective optimization method to optimize the target curve, as shown in Figure 4 and formula (1-21).

\section{Diffset}

In the experiment, a new certain type of finite element dummy is placed in a simple back and seat. The pendulum's material is rigid, and its quality is $10 \mathrm{Kg}$, while its size is $70 \mathrm{~mm} \times 100 \mathrm{~mm} \times 111 \mathrm{~mm}$. The center of pendulum keeps at the first ribs' level, and it is also in the vertical center of dummy. Frontal crash test model of thoracic pressure is shown in Figure 2. The main structure of the dummy's chest is shown in Figure 3. The speed of pendulum impacting the new finite element dummy model is set to $8.041 \mathrm{~m} / \mathrm{s}$. It can make the peak (D) of dummy's chest compression deformation reached to $50 \mathrm{~mm}$. On the frontal crash thoracic pressure curve, the slope of the most obvious fall part is considered to be the fall slope $K_{1}$ of the curve, and the slope of the most obvious rise part is considered to be the rise slope $K_{2}$ of the curve. The thoracic pressure curve for frontal crash is shown 
in Figure 4. The evaluation index value of thoracic curve is shown in Table 1. The material's initial parameters value of the main parts of the chest is shown in Table 2. It uses "Part-Number" to distinguish the material of different parts and the different parameters of the same material. The number of material parameters is eight.

Design parameters: eight material parameters are shown in Table 2.

Design goals:

1:selecting ideal parameters

2:

1): the peak of dummy's chest compression deformation

$$
D_{\text {max }}=40 \mathrm{~mm}
$$

2) the fall slope $K_{1}$ of the curve' absolute value

$$
\left|K_{1}\right|=3
$$

3 ) the rise slope $K_{2}$ of the curve' absolute value

$$
\left|K_{2}\right|=3
$$

Table 1 chest pressure curve evaluation before optimization

\begin{tabular}{cccc}
$\begin{array}{c}\text { chest pressure curve } \\
\text { evaluation } \\
\text { value }\end{array}$ & $\mathrm{D}_{\max }$ & $\mathrm{K}_{1}$ & $\mathrm{~K}$ \\
\hline & $50 \mathrm{~mm}$ & 3.8335 & 2.10462 \\
\hline
\end{tabular}

Table 2 the initial parameter values

\begin{tabular}{ccc}
\hline name_number & Parameters_unit & values \\
\hline m1_1 & $\mathrm{E}\left(\mathrm{KN} / \mathrm{mm}^{2}\right)$ & 0.20 \\
m2_1 & $\mathrm{E}\left(\mathrm{KN} / \mathrm{mm}^{2}\right)$ & 0.05 \\
m2_2 & DAMP $\left(\mathrm{mm}^{2}\right.$ & 0.50 \\
/s) & \\
m3_1 & $\mathrm{G} 0\left(\mathrm{KN} / \mathrm{mm}^{2}\right.$ & 0.57 \\
m3_2 & G1 $\left(\mathrm{KN} / \mathrm{mm}^{2}\right)$ & $7.125 \mathrm{E}-02$ \\
m3_3 & $\mathrm{BETA}\left(\mathrm{Np} / \mathrm{m}^{2}\right.$ & 0.25 \\
m4_1 & $\mathrm{m})$ & 205 \\
\hline
\end{tabular}

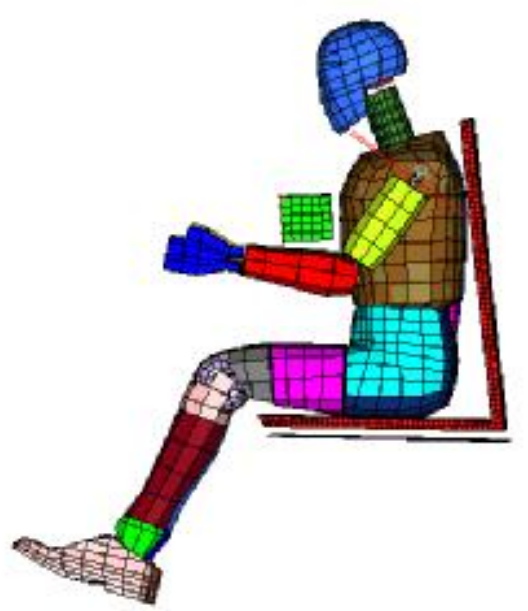

Fig.2positive collision chest pressure test 


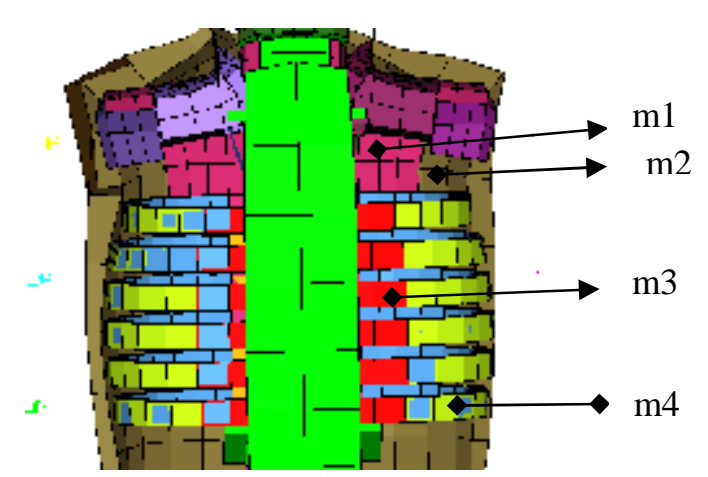

Fig.3 the dummy chest structure

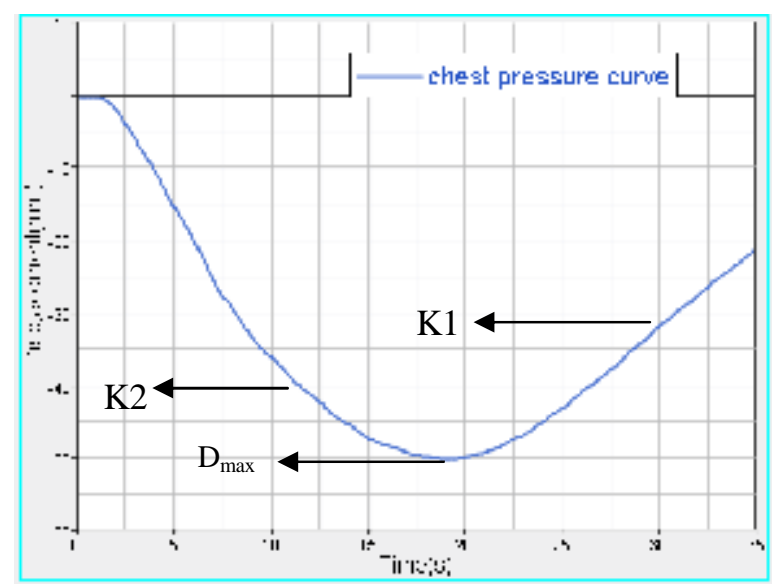

Fig 4. positive collision chest pressure curve before optimization

Table 3 optimized characteristic parameter value

\begin{tabular}{ccc}
\hline name_number & Parameters/unit & values \\
\hline $\mathrm{m} 1 \_1$ & $\mathrm{E}\left(\mathrm{KN} / \mathrm{mm}^{2}\right)$ & 0.231 \\
$\mathrm{~m} 2 \_1$ & $\mathrm{E}\left(\mathrm{KN} / \mathrm{mm}^{2}\right)$ & 0.044 \\
$\mathrm{~m} 3 \_1$ & $\mathrm{G} 0\left(\mathrm{KN} / \mathrm{mm}^{2}\right)$ & 0.372 \\
$\mathrm{~m} 3 \_2$ & $\mathrm{G} 1\left(\mathrm{KN} / \mathrm{mm}^{2}\right)$ & $6.299 \mathrm{E}-02$ \\
$\mathrm{~m} 4 \_1$ & $\mathrm{E}\left(\mathrm{KN} / \mathrm{mm}^{2}\right)$ & 173 \\
\hline
\end{tabular}

Table 4 Chest pressure curve evaluation

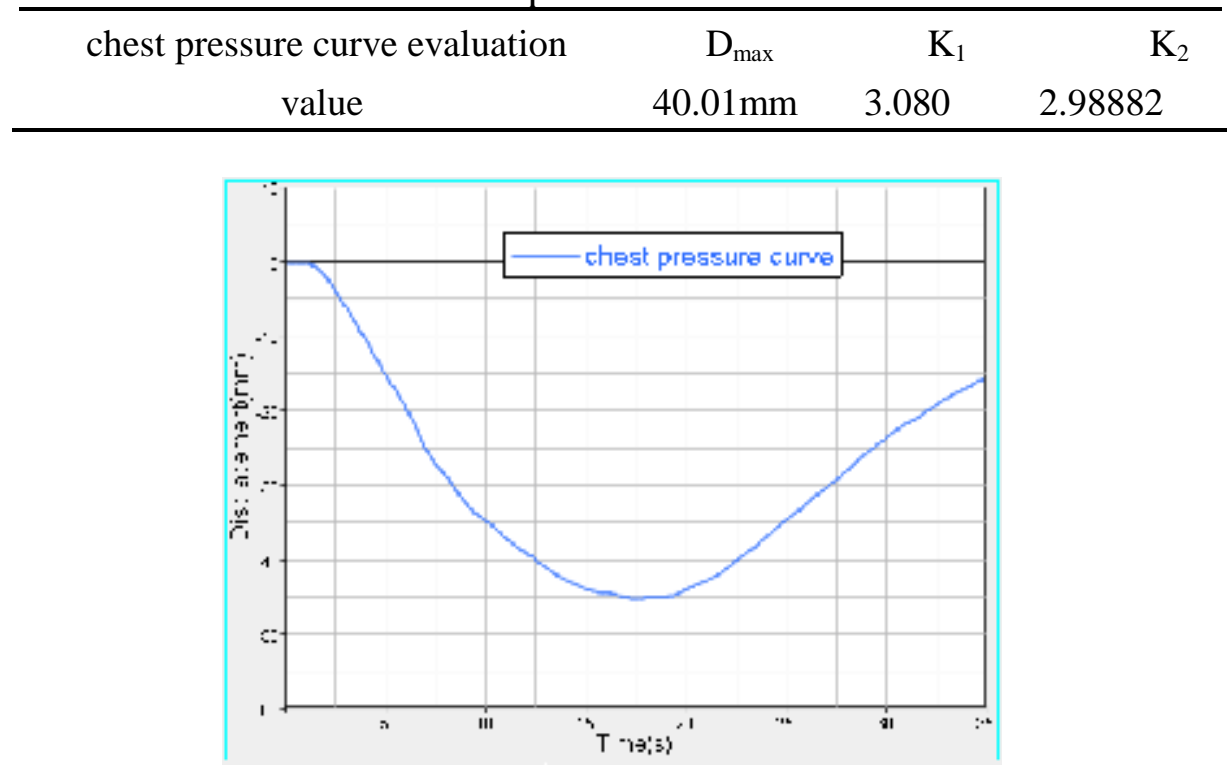

Fig.5 optimized positive collision chest pressure curve 
The dummy is optimized according to the method introduced in this paper. Select 5 useful parameters from eight known parameters as the optimization parameters through parameter optimization method, shown in table 4. Then use the multi-objective optimization method to get an optimization solution, show in table 5, and the corresponding parameters, show in table 4. Relative to the design optimization target the optimization solution have less deviation and meet the engineering design requirements.

\section{Conclusions}

In this paper, an efficient optimization method is developed based on the combination of the parameter optimization method and the multi-objective optimization method. At the same time this paper translate the curve optimization problems to the optimization of the target point of the curve which frequently meet in construction, the multi-objective optimization problem, make its optimization goal more intuitive. In this way, curve optimization problem can be simplified 、 optimization of parameters can be filtered v precision of the optimization can be guaranteed, So as to improve the efficiency of engineering optimization problems. At last, this pater's method was used in a optimization of thoracic pressure curve at a type of domestic car, further proves the feasibility of this method.

\section{Acknowledgements}

This work was financially supported by the Jilin Province Science and Technology Department Yout h Researvh Foundation (20130522180JH) and the High-end Technology Innovation Platform of Aut omobile Parts Manufacturing Technology and Equipment in Colleges and Universities in Jilin Provi nce(440020031101)。

\section{References}

[1] China Automotive Technology \&Research Center. C-NCAP China-New Car Assessment Program. [S].2006.

[2] Hao Ting, Wang Yong. Application analysis of vehicle crash test and hybrid IIIdummy [J]. Urban Vehicles. 2003,1:21-23

[3] Wang Tao, Tao Wei. Multi-objective Robust Optimization of Automobile Suspension Parameters Considering Random Factors [J].Journal of Vibration and Shock，2009，28(11):146-149.

[4] Wang Tao. Multi-objective and Multi-criteria Decision Optimization of Automobile Suspension Parameters[J]. Journal of agricultural machinery, 2009,40 (4):27-32.

[5] Wang Tao, Tao Wei. Multi-objective optimization of heavy truck suspension parameters [J].Modular Machine TOOL \& Automatic Manufacturing Technique, 2008(11):29-31.

[6] Guan Xin, Feng Shu-Yi, Zhan Jun. Optimization of suspension Kinematics characteristic parameters[J]. Journal of Harbin institute of technology, 2011,43 (1) :145-148.

[7] Ma Kai, Chen Su-Huan. Iterative approximation algorithm of Hessian matrix in structural optimization [J]. Journal of Jilin University (Engineering and Technology Edition), 2006, 36 (1) :30-33.

[8] Chen Su-Huan , Ma, Kai .Combined optimization method of plate-shell structures with stiffeners[J]. Journal of Northeastern University(Natural Science). 2008， S (2):400-405. 
[9] Ma Kai, Chen Su-huan. Approximation algorithm of Hessian matrix in interval optimization of dynamic analysis for structures with interval parameters[J] Journal of Northeastern University, 2008,38(Sup.2):221-224.

[10] Wu Jie, Shangguan Wen-bin, Chen Su-huan. Improved dynamic optimization method for uncertain structures with interval parameters[J].Noise and Vibration Control, 2008, 28(1):27-32.

[11] Chen Su-huan, Wu Jie, Chen Yu-dong. Interval optimization for uncertain structures[J].Finite Elements in Analysis and Design,2004,40:1379-1398.

[12] Chen Su-huan, Wu Jie, Interval optimization of dynamic response for uncertain structures with natural frequency constraints [J].Engineering Structures, 2004, 26:221-232.

[13] Ma Kai, Guan Xin, Li Peng. Parameter Selection Method in Optimization of Suspension Kinematics Characteristics[J]. Automotive Engineering, 2013,35(6):517-520.

[14] Chen Hong-Yu, Ma Kai. The Interval Analysis Method on Coherence of Automobile Suspension $\mathrm{K} \& \mathrm{C}[\mathrm{J}]$.Value Engineering, 2012,32:0001-03.

[15] Chen Su-Huan. The Matrix Perturbation Theory of Structural Vibration Analysis. [M].Chongqing: Chongqing Publishing House.1991.

[16]Ma Kai, Guan Xin, Feng Shu-Yi, Zhan Jun. Interval Control Method on Consistency of Suspension Kinematics[J]. Journal of Jilin University (Engineering and Technology Edition), 2011, 41 (04): :910-914.

[17]Ma Kai, Feng Shu-Yi, Zhan Jun, Guan Xin. Second-order Approximation Interval Optimization of Suspension Kinematic Characteristics[J]. Automotive Engineering, 2011,33(6):527-530. 